\title{
Formulation and Evaluation of Gastro-bilayer Floating Tablets of Simvastatin as Immediate Release Layer and Atenolol as Sustained Release Layer
}

\author{
R. P. SWAIN*, SHILPA PENDELA AND S. PANDA
}

Department of Pharmaceutical Technology, Maharajah's College of Pharmacy, Vizianagaram-535 002 , India

Swain, et al.: Gastro-bilayer Floating Tablets of Simvastatin and Atenolol

\begin{abstract}
Combination therapy is highly desired to deliver atenolol and simvastatin for dyslipidemia and hypertension as a concomitant drug treatment. On other hand, atenolol has poor absorption in lower gastrointestinal tract, short half-life and simvastatin has poor solubility. Therefore, the present study was to develop gastro-bilayer floating matrix tablet in which simvastatin was incorporated as immediate layer and atenolol as sustained release layer. Gastro-bilayer floating tablets were prepared by direct compression method and optimized using hydroxypropyl methylcellulose $\mathrm{K} 100(37.5 \% \mathrm{w} / \mathrm{w})$ as release retardant and sodium bicarbonate as a gas-forming agent. The immediate release layer comprised sodium starch glycolate as superdisintegrant. The tablets of optimized formulations floated on the test medium for more than $12 \mathrm{~h}$ with 9 min of floating lag time. Atenolol release was sustained for $12 \mathrm{~h}$ via diffusion mechanism and more than $96 \%$ release of simvastatin within 15 min was achieved. It can be concluded that, the biphasic drug release pattern was successfully achieved through the formulation of gastro-floating bilayer tablets in this study, allowing strengthened combination therapy for hypertension and dyslipidemia.
\end{abstract}

Key words: Simvastatin, atenolol, gastro-bilayer, floating, immediate release, sustained release

Oral drug delivery is most desirable route of administration because of its high patient satisfactoriness,flexibility in formulation and stability ${ }^{[1]}$. Factors that often impact the absorption of orally administered drugs include frequent dosing which results in fluctuation of plasma drug concentration and finally toxicity ${ }^{[2]}$. To surmount this problem oral controlled drug delivery system that delivers drug for an extended period of time was developed. This system has many difficulties due to physiological problems like drugs with narrow absorption window, alteration in emptying time of stomach, drugs that have stability issues in intestine and drugs that are transported via active transport mechanism ${ }^{[3]}$. To overcome these difficulties gastroretentive drug delivery system (GDDS) has been developed. Gastroretentive systems can remain in the gastric cavity for several hours and prolong the gastric residence time of drugs. Prolonged gastric retention improves bioavailability, reduces drug waste, and improves solubility for drugs that are less soluble in a high $\mathrm{pH}$ environment. It also provides local drug delivery to the stomach and proximal small intestine ${ }^{[4]}$. Among the gastroretentive dosage forms,

*Address for correspondence

E-mail: ranjit.prasad797@gmail.com

July-August 2016 floating drug delivery system is considered to be most favourable because it does not intensely affect the motility of gastrointestinal tract (GIT) ${ }^{[5]}$. The matrix bilayer tablet with two separate release-layers is a biphasic delivery system that aims to deliver drug at two different rates or simultaneously releases two drugs with the following benefits, formulating two chemically incompatible drugs into a system, simultaneously releasing two active pharmaceutical ingredients (APIs) with desired release profiles, increasing efficacy of API by a synergistic effect, decreasing the dosing unit burden and better patient compliance ${ }^{[6]}$.

Cardiovascular disease is a collective term that covers any disease of heart and circulatory system $^{[7]}$. The foremost risk factors for cardiovascular diseases are tobacco exposure, hypertension, high cholesterol,

This is an open access article distributed under terms of the Creative Commons Attribution-NonCommercial-ShareAlike 3.0 License, which allows other the remix, tweak, and build up to the non-commercially, as long as the author is credited and the new creations are licensed under the identical terms.

Accepted 10 July 2016

Revised 24 March 2016

Received 06 January 2016

Indian J Pharm Sci 2016;78(4):458-468 
alcohol consumption and obesity ${ }^{[8]}$. Globally cardiovascular diseases account for approximately 17 million deaths each year ${ }^{[9]}$ of which 9.4 million deaths are due to hypertension ${ }^{[10]}$. The first National Health and Nutrition Examination Survey (NHANES) estimated the coprevalence of hypertension and elevated total cholesterol. During the third NHANES, around $60 \%$ of hypertensive adults had concomitant hypercholesterolemia and $55 \%$ of the adults with hypercholesterolemia had hypertension ${ }^{[11]}$. Therefore, hypertension and hypercholesterolemia often coexist and may require concomitant drug treatment ${ }^{[12]}$.

Atenolol, a cardioselective beta-1 adrenoceptor is devoid of intrinsic sympathomimetic and membrane stabilizing activity ${ }^{[13]}$. It belongs to the category of antihypertensive agents and helps in reducing high blood pressure. Atenolol is also included in the treatment of angina and cardiac arrhythmia ${ }^{[14]}$. It has absorption window in the upper GIT whereas the poor absorption in lower GIT. This varied absorption results in lowering bioavailability i.e. $50 \%$ with a halflife of $6-8 \mathrm{~h}^{[15]}$. Thus it seems that increase in gastric residence time may increase the extent of absorption and bioavailability of drug. Therefore atenolol was incorporated into the sustained release layer to improve its bioavailability. Simvastatin is classified under antihyperlipidemics (statins) and mainly acts by inhibiting HMG Co-A reductase inhibitor ${ }^{[16]}$. It is a BCS class II drug with a half-life of less than $3 \mathrm{~h}$ and bioavailability of less than $5 \%$ as its absorption is limited by its release rate ${ }^{[17]}$. Accordingly, the solubility of simvastatin has been improved and formulated into immediate release layer to achieve better absorption.

Kulkarni et al. worked on development and evaluation of bilayer floating tablets of atenolol and lovastatin for biphasic release profile to improve the bioavailability of atenolol and to treat hyperlipidemia which is the major problem in hypertensive patients ${ }^{[18]}$. Another researcher also worked on gastro-floating bilayer tablets, formulated them for the sustained release of metformin and immediate release of pioglitazone, as metformin has poor absorption in colon and pioglitazone reduces the risk of atherosclerosis, a major problem in diabetic patients $^{[5]}$.

Combination therapies that target several risk factors, such as hypercholesterolemia, hypertension, diabetes and artery function may more effectively treat cardiovascular disease than a therapy that targets a single risk factor. Currently high cholesterol is a major risk factor for the cardiovascular death. So combination therapy is required to deliver drugs for dyslipidemia and hypertension. To the best of our knowledge, no reported literature was found on the combination therapy of simvastatin and atenolol. In the present study we have made an effort to formulate a gastrobilayer floating system of simvastatin in the immediate release layer and atenolol in the sustained release layer to improve their bioavailability.

\section{MATERIALS AND METHODS}

Atenolol was procured from Yarrow Chem Products (Mumbai, India). Simvastatin was a gift sample from National Chemicals (Vadodara, India). Hydroxypropyl methylcellulose and poloxamer 188 (p 188) were supplied by Piramal Health Care (Bengaluru, India). PEG 6000 and HP $\beta$-cyclodextrin (HP- $\beta$-CD) were purchased from Molychem (Mumbai, India). Sodium bicarbonate, sodium starch glycolate and lactose were obtained from Thermo Fischer Scientific Pvt. Ltd (Ahmedabad, India). Microcrystalline cellulose was obtained from HiMedia (Gurgaon, India). Ethanol and $\mathrm{HCl}$ were of analytical grade.

\section{Preparation of immediate release layer:}

Solubility of simvastatin was improved by preparing three formulations $\mathrm{CM} 1, \mathrm{CM} 2, \mathrm{CM} 3$ with three different carries PEG 6000, HP- $\beta-C D$, p 188 in the drug: polymer ratio of 1:5 using cogrinding technique. All prepared formulations were passed through sieve $44 \#$ to get uniform size and kept in desiccators for further use ${ }^{[19-21]}$.

\section{Characterization of cogrinding mixture:}

FTIR Spectra of simvastatin, p 188 and cogrinding mixture were subjected to compatibility studies by compressing with $\mathrm{KBr}$ at 20 psi for 10 min to get pellet. On $\mathrm{KBr}$ pellet the spectra were scanned in the wave number range of 4000-600 $\mathrm{cm}^{-1}$.

\section{Differential scanning calorimetry (DSC):}

Thermal analysis was carried out for simvastatin, $\mathrm{p} 188$ and cogrinding mixture using DSC (Pyris Diamond, Singapore). The samples were heated at a constant rate of $10^{\circ} / \mathrm{min}$ over a temperature range of $30-280^{\circ}$ using platinum crucible with alpha alumina powder as reference to predict any physicochemical interactions between components.

\section{X-ray diffraction analysis (XRD):}

Simvastatin, p 188 and cogrinding mixture were subjected to XRD analysis, using $\mathrm{Cu}$ target slit $10 \mathrm{~mm}$ (ULTIMA III, Japan) to investigate physical state of simvastatin, $\mathrm{p} 188$ and simvastatin cogrinding mixtures. 


\section{In vitro dissolution studies:}

Dissolution studies were performed for the cogrinding mixtures using USP dissolution type II apparatus (paddle method) with $50 \mathrm{rpm}$ in $900 \mathrm{ml}$ of $0.1 \mathrm{M} \mathrm{HCl}$ as dissolution medium at $37 \pm 0.5^{\circ}$. The samples were withdrawn at predetermined time intervals $(5,10,15$, 30,45 and $60 \mathrm{~min}$ ) and the same amount of preheated $\left(37 \pm 0.5^{\circ}\right)$ fresh medium was added to maintain constant volume throughout study. The percentage drug release values obtained from the dissolution studies were plotted against time in $\mathrm{h}$.

\section{Compression of immediate release tablets:}

Cogrinding mixture equivalent to $10 \mathrm{mg}$ of simvastatin was blended with sodium starch glycolate, lactose to prepare IF1, IF2, IF3 formulations and passed through sieve $44 \#$ then add magnesium stearate before direct compression of tablets (Table 1).

\section{Compression of sustained release tablets:}

All the ingredients of formulations (SF1-SF9) shown in Table 2 were accurately weighed and sifted through sieve 44\# to obtain uniform size. Magnesium stearate was added before punching of tablets by direct compression method.

\section{Preparation of gastro-bilayer floating tablets:}

Based on the dissolution profiles of immediate release tablets (IF3) and sustained release tablets (SF9), the gastro-bilayer floating tablets were prepared with varying concentration of sodium bicarbonate in the sustained release layer (Table 3). Bilayer floating tablets were fabricated with multi station rotary tablet punching machine via single compaction method. Initially sustained release layer powder blend was accurately weighed and fed into die cavity of tablet punching machine. Similarly immediate release layer powder was fed to die cavity which was previously filled with the sustained release layer and compressed so that the final hardness obtained for a bilayer floating tablet was between $5.5-6.5 \mathrm{~kg} / \mathrm{cm}^{2}$ using $12 \mathrm{~mm}$ flat punches.

\section{Post compression parameters of gastro-bilayer} floating tablets:

Thickness of the ten randomly selected tablets from each batch was determined with Vernier caliper scale (Model: 530-312, Japan).

\section{Weight variation:}

Twenty tablets were randomly selected from each batch and calculated the percentage deviation of individual tablet weight from average weight of tablets.

\section{Crushing strength:}

Crushing strength was determined using Monsanto type hardness tester by selecting randomly six tablets from each batch.

\section{Friability:}

Friability test was conducted by considering tablets

TABLE 1: FORMULATION OF IMMEDIATE RELEASE LAYER BY DIRECT COMPRESSION METHOD

\begin{tabular}{cccccc}
\hline $\begin{array}{c}\text { Formulation } \\
\text { code }\end{array}$ & $\begin{array}{c}\text { Cogrinding mixture equivalent to } \\
\text { mg of simvastatin }\end{array}$ & SSG (mg) & Lactose $(\mathrm{mg})$ & $\begin{array}{c}\text { Magnesium } \\
\text { stearate }(\mathbf{m g})\end{array}$ & Total (mg) \\
\hline IF1 & 60 & 5 & 34 & 1 & 100 \\
IF2 & 60 & 10 & 29 & 1 & 100 \\
IF3 & 60 & 15 & 24 & 1 & 100 \\
\hline
\end{tabular}

Each batch contains 10 tablets

TABLE 2: FORMULATION OF SUSTAINED RELEASE LAYER BY DIRECT COMPRESSION METHOD

\begin{tabular}{cccccccc}
\hline $\begin{array}{c}\text { Formulation } \\
\text { code }\end{array}$ & Drug $(\mathbf{m g})$ & $\begin{array}{c}\text { HPMC K4 } \\
(\mathbf{m g})\end{array}$ & $\begin{array}{c}\text { HPMC K15 } \\
(\mathbf{m g})\end{array}$ & $\begin{array}{c}\text { HPMC K100 } \\
(\mathbf{m g})\end{array}$ & MCC (mg) & $\begin{array}{c}\text { Magnesium } \\
\text { stearate }(\mathbf{m g})\end{array}$ & Total (mg) \\
\hline SF1 & 50 & 50 & - & - & 292 & 8 & 400 \\
SF2 & 50 & 100 & - & - & 242 & 8 & 400 \\
SF3 & 50 & 150 & - & - & 192 & 8 & 400 \\
SF4 & 50 & - & 50 & - & 292 & 8 & 400 \\
SF5 & 50 & - & 100 & - & 242 & 8 & 400 \\
SF6 & 50 & - & 150 & - & 192 & 8 & 400 \\
SF7 & 50 & - & - & 50 & 292 & 8 & 400 \\
SF8 & 50 & - & - & 100 & 242 & 8 & 400 \\
SF9 & 50 & - & - & 150 & 192 & 8 & 400 \\
\hline
\end{tabular}

Each batch contains 10 tablets 
TABLE 3: FORMULATION OF GASTRO-BILAYER FLOATING TABLETS

\begin{tabular}{lccc}
\hline Ingredients (mg) & ISF1 & ISF2 & ISF3 \\
\hline Immediate release layer & & & \\
Cogrinding mixture equivalent to & 60 & 60 & 60 \\
10 mg of simvastatin & & & \\
SSG & 15 & 15 & 15 \\
Lactose & 24 & 24 & 24 \\
Magnesium stearate & 1 & 1 & 1 \\
Sustained release layer & & & \\
Atenolol & 50 & 50 & 50 \\
Sodium bicarbonate & 15 & 20 & 25 \\
MCC & 177 & 172 & 167 \\
Magnesium stearate & 8 & 8 & 8 \\
Total & 500 & 500 & 500 \\
\hline
\end{tabular}

Values are in $\mathrm{mg}$, each batch contains 50 tablets

whose weight equivalent to $6.5 \mathrm{~g}$ using Roche friabilator apparatus (Model: 40 FT A01). It was rotated for $4 \mathrm{~min}$ at $25 \mathrm{rpm}$ where the tablets were allowed to fall from 6 inches height in each turn within the apparatus later these tablets were weighed to calculate percentage friability.

\section{Drug content:}

Twenty tablets were randomly selected and crushed for the estimation of drug content. Powder weight equivalent to $50 \mathrm{mg}$ was transferred into $50 \mathrm{ml}$ volumetric flask and made to the volume by 0.1 $\mathrm{M} \mathrm{HCl}$. The flask was placed in a sonicator till completely soluble. The solution was filtered through a filter paper $(0.45 \mu \mathrm{m}$ pore size $)$ and from this $1 \mathrm{ml}$ was taken and transferred to $25 \mathrm{ml}$ volumetric flask which was made up to the mark by $0.1 \mathrm{M} \mathrm{HCl}$. The absorbance of the solution was measured using UV/ Vis spectrophotometer (Agilent, Cary 60) against the blank $0.1 \mathrm{M} \mathrm{HCl}$ at 240 and $225 \mathrm{~nm}$ for estimation of simvastatin and atenolol, respectively.

\section{In vitro floating ability:}

The buoyancy capability of floating tablets was visually determined by employing the method described by Rosa et al. ${ }^{[22]}$ Buoyancy test was carried out by placing the tablet in beaker containing $250 \mathrm{ml}$ of $0.1 \mathrm{M} \mathrm{HCl}$. The time required for floating tablets to appear on to the surface of a dissolution medium (floating lag time) and the time during which the dosage form constantly float (total float time) were measured.

\section{Dissolution studies of gastro-bilayer floating tablets:}

Dissolution study was carried out by operating USP type II apparatus (paddle method) at a rotational speed of $50 \mathrm{rpm}$ with $900 \mathrm{ml}$ of $0.1 \mathrm{M} \mathrm{HCl}$ dissolution medium that was maintained at $37 \pm 0.5^{\circ}$ throughout the study. At predetermined time intervals samples were withdrawn for a period of $12 \mathrm{~h}$. To maintain constant volume, $5 \mathrm{ml}$ of sample was withdrawn and replaced with equal volume of fresh medium maintained at $37 \pm 0.5^{\circ}$ and the samples were filtered through filter paper $(0.45 \mu \mathrm{m}$ pore size). The samples withdrawn were analyzed by using a UV/Vis spectrophotometer at $240 \mathrm{~nm}$ and $225 \mathrm{~nm}$ for the estimation of simvastatin and atenolol, respectively.

\section{Release kinetics $^{[23-25]}$ :}

In vitro drug release data was subjected to mathematical models like zero order, first order, Higuchi, Hixson Crowell, Korsmeyer and Peppas in order to investigate the release pattern of formulated batches.

\section{RESULTS AND DISCUSSION}

Simvastatin is a BCS class II drug which is practically insoluble in water $(0.0122 \mathrm{mg} / \mathrm{ml})$. So, in order to meet the aim of the study, an attempt was made to improve the solubility of simvastatin. Therefore for improving solubility, we prepared three formulations CM1, CM2, CM3 with three different carriers PEG 6000, HP- $\beta-C D$, p 188 by adopting cogrinding technique in the drug: polymer ratio of 1:5 and subjected for physicochemical characterization like FTIR, DSC, XRD, SEM and in vitro dissolution studies.

FTIR spectrum of pure drug simvastatin was characterized by $-\mathrm{OH}$ stretching vibrations at 3550.20 $\mathrm{cm}^{-1}$, methyl $\mathrm{C}-\mathrm{H}$ asymmetric stretching at 3011.38 $\mathrm{cm}^{-1}$, ester $\mathrm{C}=\mathrm{O}$ stretching at $1699.66 \mathrm{~cm}^{-1}$ and lactone $-\mathrm{C}-\mathrm{O}-\mathrm{C}$ stretch at $1268.27 \mathrm{~cm}^{-1}$. The results were in agreement with Pandya et al. ${ }^{[26]}$. FTIR spectrum of cogrinding mixture showed peak values at 2885.80, $1343.85,1112.05,842.58 \mathrm{~cm}^{-1}$ which showed that the intensities of these peaks were reduced in the cogrinding mixture and slight shift in the peak values indicates, the carrier and simvastatin were sufficiently mixed without any chemical interactions (fig. 1).

DSC thermogram of pure drug simvastatin showed endotherm peak at $138.78^{\circ}$ corresponds to its melting point. No melting endotherm of solid dispersions corresponding to pure drug simvastatin was observed but it showed the peak at lower temperature i.e. $58.35^{\circ}$ corresponding to the melting peak of $\mathrm{p} 1888$ (fig. 2). From this, we concluded that simvastatin was completely dispersed in the carrier. 


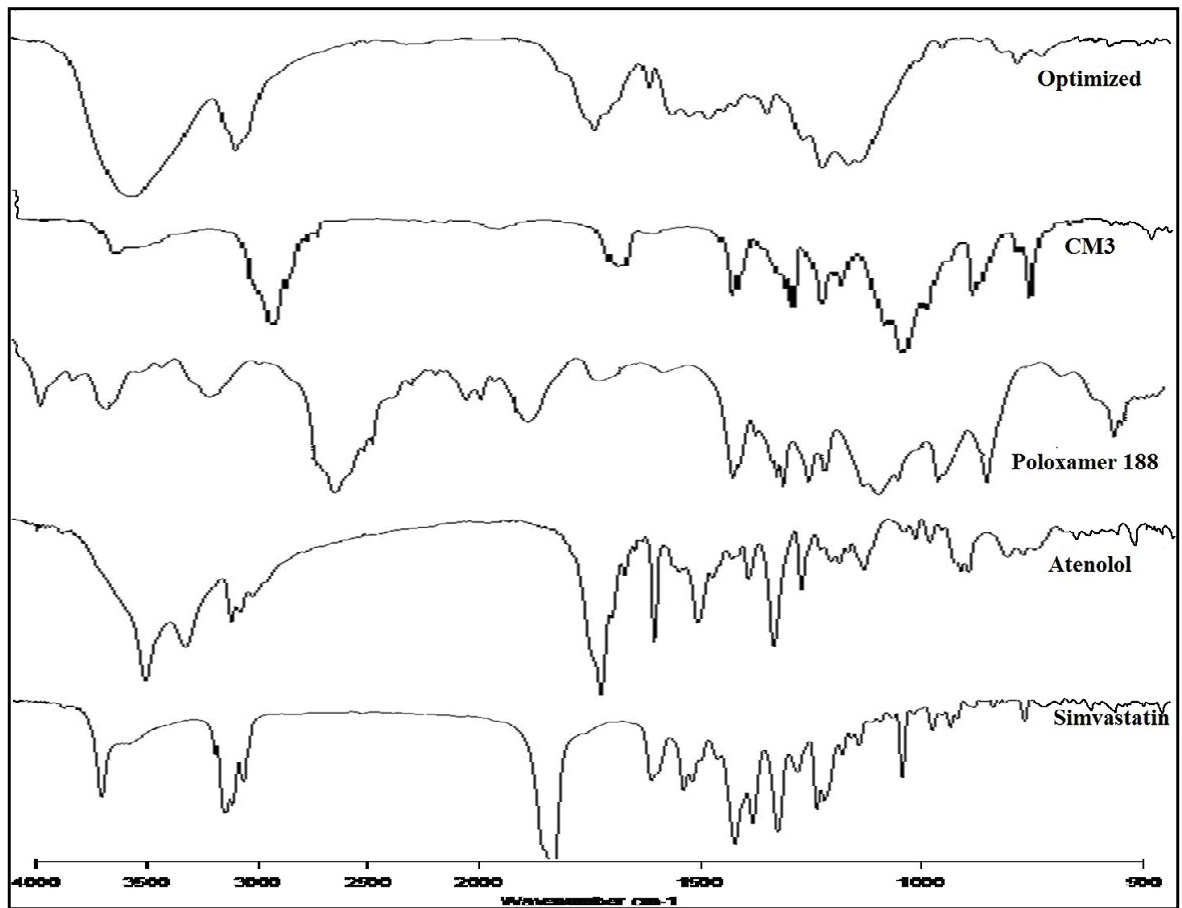

Fig. 1: FTIR absorption spectra.

FTIR absorption spectra of simvastatin, atenolol, p 188, cogrinding mixture (CM3) and optimized bilayer tablet.

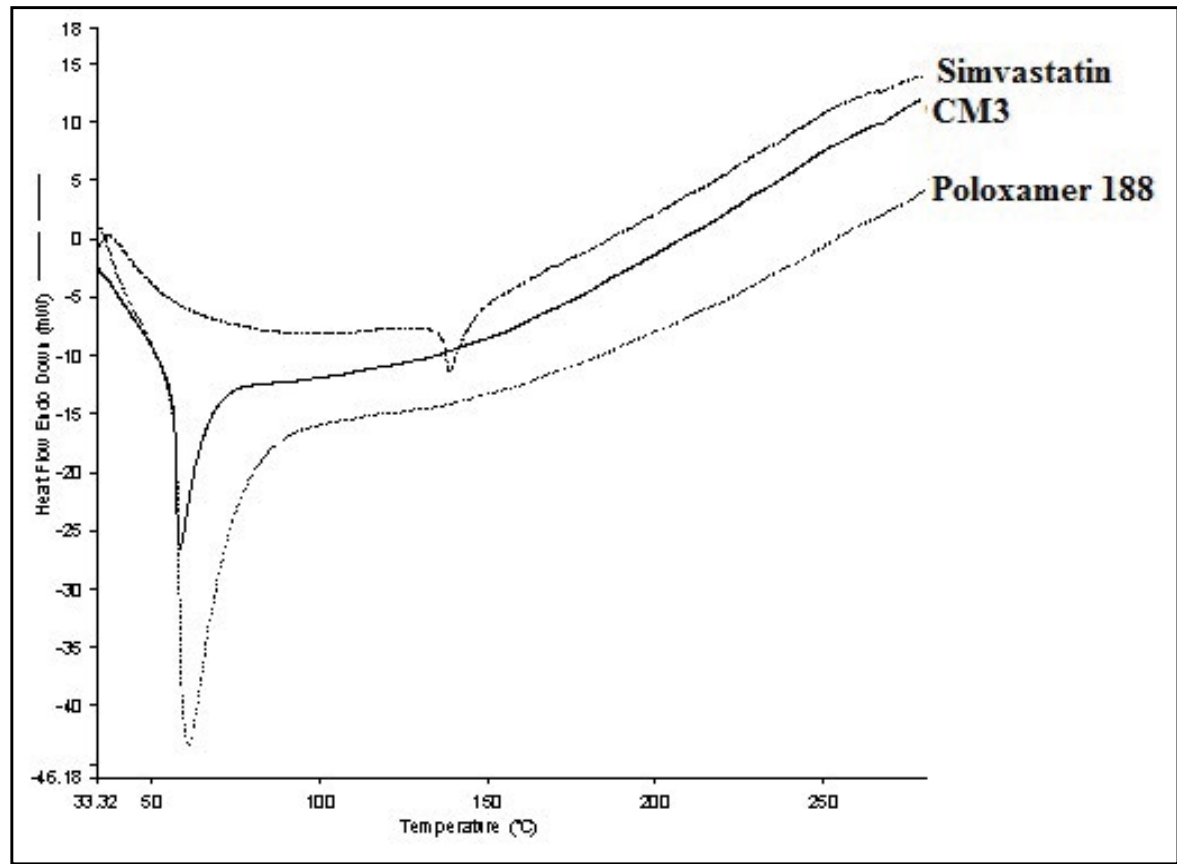

Fig. 2: DSC thermograms.

DSC thermograms of simvastatin, $p 188$ and cogrinding mixture (CM3).

The XRD patterns of pure drug simvastatin, $\mathrm{p} 188$, CM3 were shown in fig. 3. In the X-ray diffractogram of pure drug simvastatin, sharp peaks were detected at $2 \theta$ equivalent to $9^{\circ}, 11^{\circ}, 17^{\circ}, 19^{\circ}, 23^{\circ}$ showed crystalline nature of simvastatin. In the cogrinding mixture of simvastatin, XRD showed no sharp peaks at $2 \theta$ that indicates crystalline nature of simvastatin was converted to amorphous form. Peaks detected in the XRD of cogrinding mixture might be due to $\mathrm{p} 188$.

The dissolution profiles obtained for pure drug simvastatin and cogrinding mixtures of simvastatin prepared in drug: carrier ratio by using various carriers were shown in the fig. 4. The dissolution studies revealed that there was notable difference 


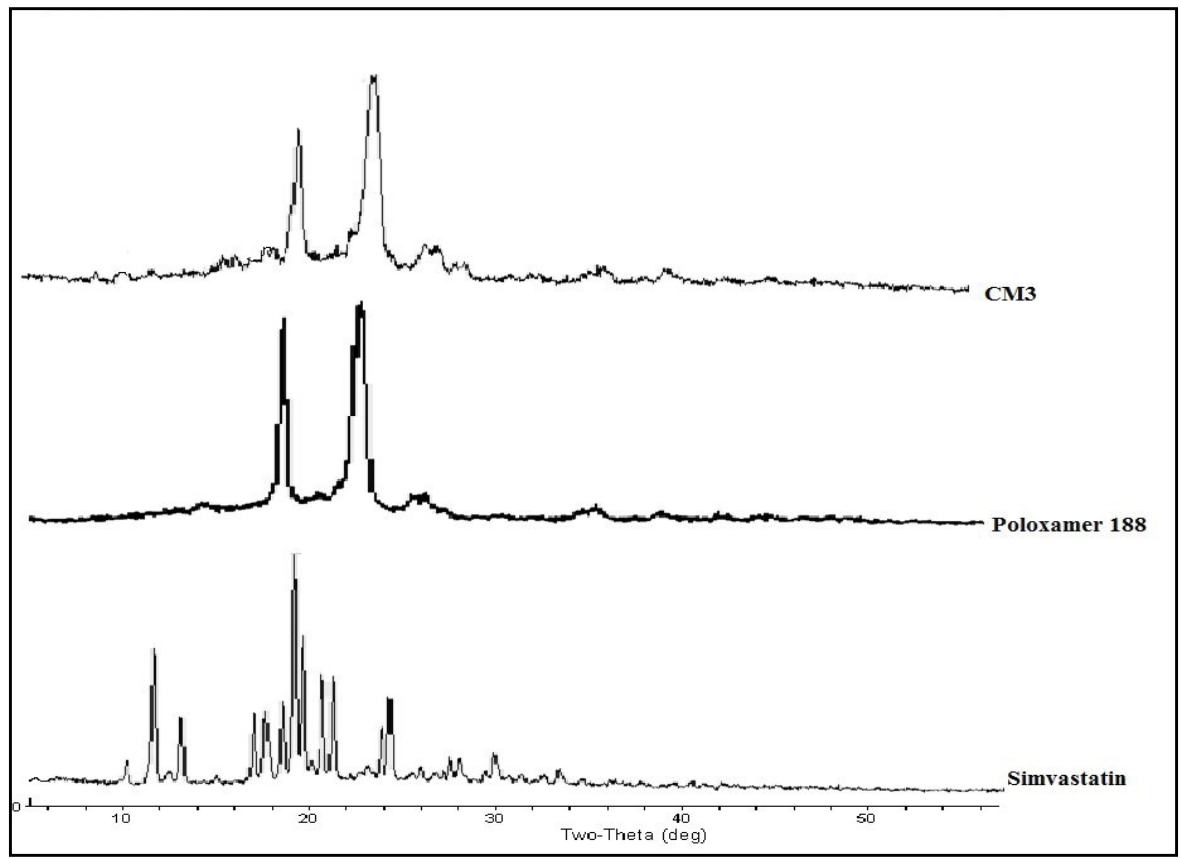

Fig. 3: XRD pattern.

XRD pattern of simvastatin, $p 188$ and cogrinding mixture (CM3).

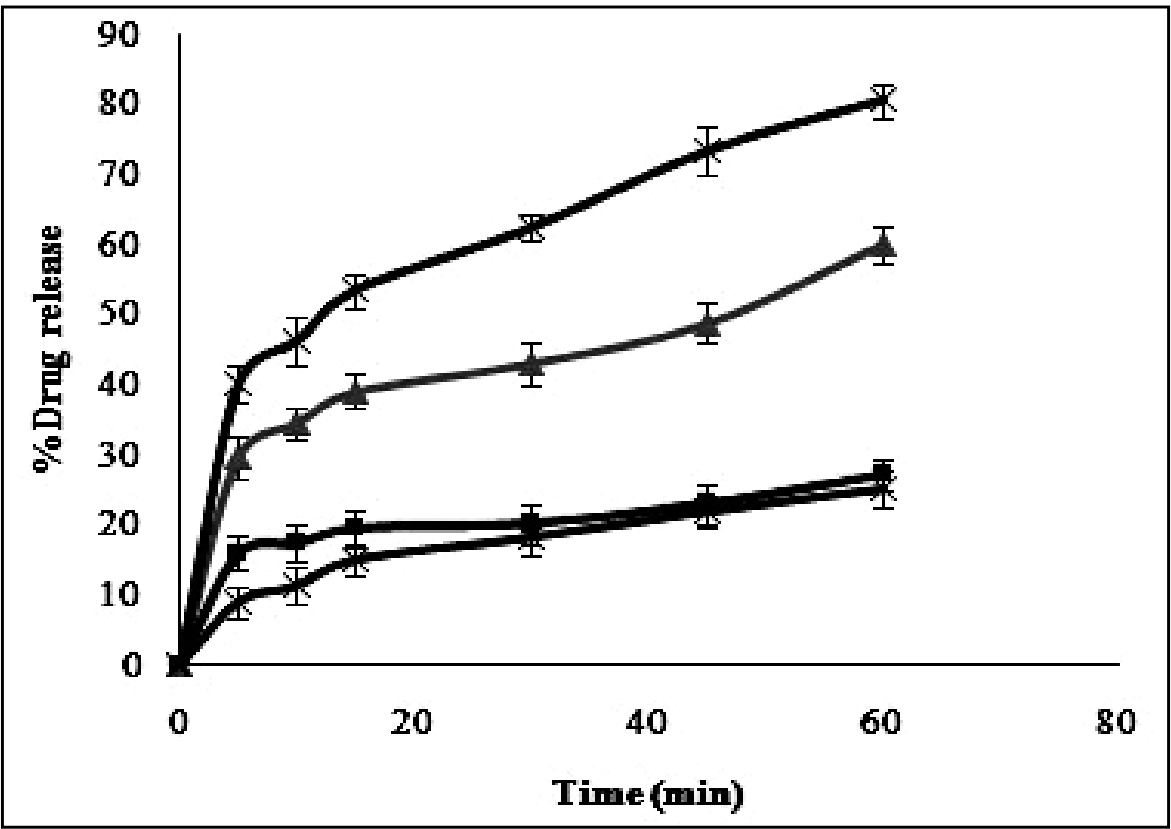

Fig. 4: In vitro release profiles for solubility enhancement of simvastatin.

Pure drug (-*-); CM1 (-口-); CM2 (- - -); CM3 (-×-)

between dissolution profile of pure drug simvastatin and simvastatin with carriers. The dissolution profile values obtained for simvastatin with $\mathrm{p} 188$ in the ratio of 1:5 (CM3) showed higher dissolution rate i.e. $80 \%$ in $1 \mathrm{~h}$ than the formulations prepared with $\mathrm{HP}-\beta-\mathrm{CD}$ and PEG 6000. Formulation CM3 (p 188) showed a 3 fold increase in solubility when compared to the pure drug. Solubility of simvastatin might be enhanced due to amphiphilic nature of $\mathrm{p} 188$ that helps in reducing the contact angle between drug and dissolution medium there by increasing the wettability of drug, also reduction in crystallinity of drug led to decrease of the energy required in the dissolving process which was supported by XRD and DSC. The formulated batch, CM3 equivalent to $10 \mathrm{mg}$ of simvastatin was taken and formulated into immediate release tablet batches (IF1, IF2, IF3) by varying the concentration of superdisintegrant, sodium starch glycolate and following direct compression method. Dissolution profiles of the prepared formulations were depicted 
in fig. 5. The formulation containing $15 \% \mathrm{w} / \mathrm{w}$ of sodium starch glycolate showed $98.8 \%$ of drug release in $15 \mathrm{~min}$. This shows 4 fold increase in dissolution efficiency when compared to the pure drug over a period of $1 \mathrm{~h}$. This may be due to when the formulation comes in contact with the dissolution medium, sodium starch glycolate in the immediate release tablets swells by absorbing the liquid medium thereby liberating simvastatin with fine dispersion. Therefore, IF3 formulation was selected as immediate release layer in the preparation of gastro-bilayer floating tablets.

Atenolol is a class III drug with high solubility and low permeability with a half-life of 6-7 $\mathrm{h}$ and therefore, polymer should be incorporated into the formulation to retard the release. HPMC is a hydrophilic polymer and is strong enough to retard the release depending upon its viscosity and concentration ${ }^{[27]}$. Formulations with three different grades of HPMC (K4, K15, and $\mathrm{K} 100)$ in three different concentrations $(12.5,25$, and $37.5 \% \mathrm{w} / \mathrm{w}$ ) including all other ingredients were accurately weighed and these powder blends were used for the preparation of sustained release tablets by direct compression method. The release profiles of entire formulation batches SF1-SF9 were illustrated in fig. 6. Hydrophilic nature of HPMC facilitates penetration of dissolution medium into the network structure of polymer chain thereby causing hydration of the polymer hence swelling ${ }^{[18]}$. Gelatinous layer may act as boundary for the drug that has to be released.
The formulations with HPMC K100 (SF7, SF8, SF9) showed $99.9 \%$ of drug release within $12 \mathrm{~h}$ whereas HPMC K15 (SF4, SF5, SF6) and HPMC K4 (SF1, SF2, SF3) showed $99 \%$ of drug release in less than 7 and $5 \mathrm{~h}$ respectively. This showed that HPMC K4 and HPMC K15 polymers were unable to maintain their integrity for longer period when compared to HPMC K100. Therefore HPMC K100 showed better retardant effect when compared to other two polymers (HPMC $\mathrm{K} 4$ and HPMC K15) over a period of $12 \mathrm{~h}$. Among three different concentrations of HPMC K100 the formulation with $37.5 \% \mathrm{w} / \mathrm{w}$ of polymer (SF9) showed good release profile over a period of $12 \mathrm{~h}$. This may be due to increased concentration of polymer that increase the thickness of gel barrier and tortuosity and also it follows release profile as per Robinson and Eriksen equation i.e. $30-35 \%$ of drug released in $1 \mathrm{~h}$ and $60-$ $65 \%$ of the drug released after $6 \mathrm{~h}$ and the remaining drug released after $12 \mathrm{~h}^{[13]}$. Therefore SF9 is selected as the optimized formulation and as sustained release layer in the preparation of gastro-bilayer floating tablets.

Thickness depends on the quantity of powder blend (bulk density). It evaluates the uniformity in hardness of prepared tablets. There was no much variation (2.48$2.43 \mathrm{~mm}$ ) in thickness of tablets in each formulation that showed the powder blends were consistent in particle size, size distribution and facilitates packaging.

Weight variation test was performed to check uniformity

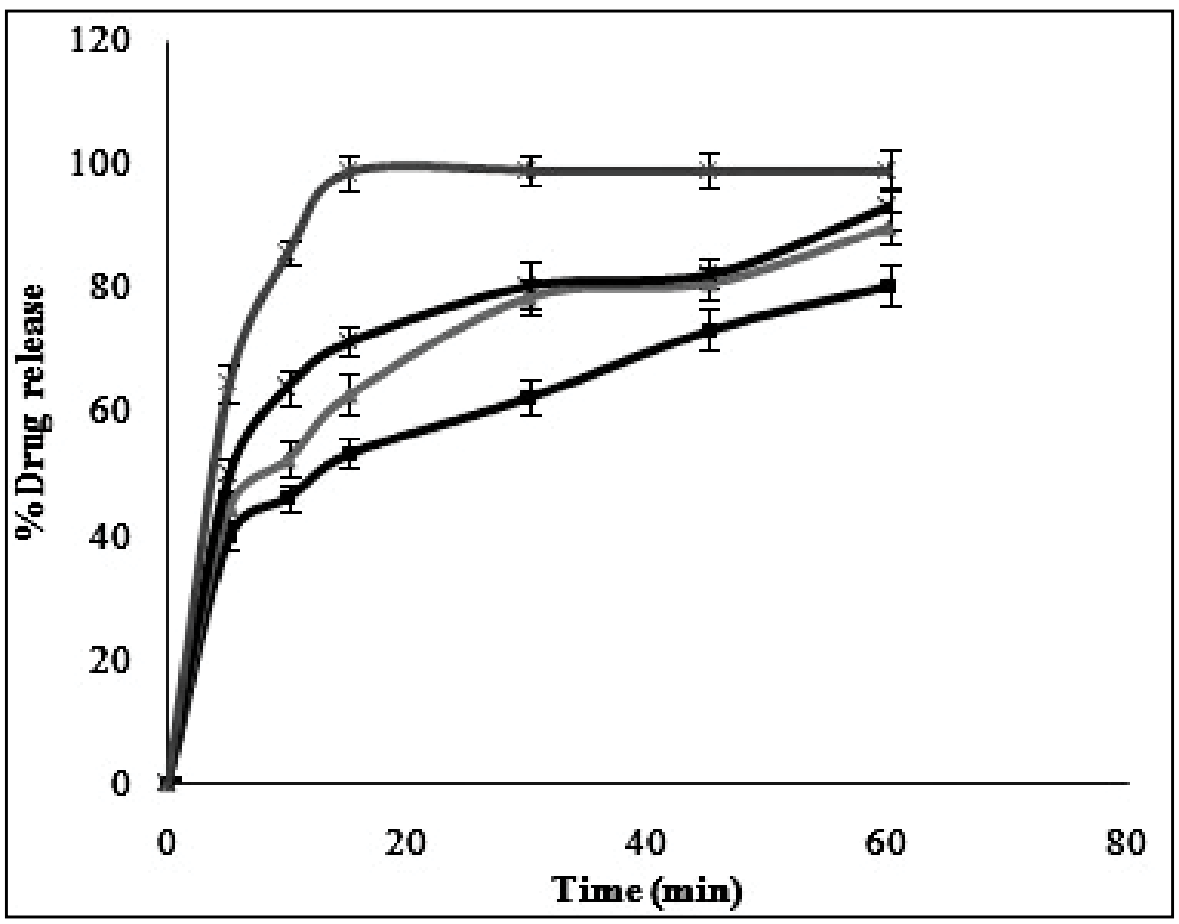

Fig. 5: In vitro release profiles of immediate release tablets.

Optimized cogrinding mixture (-匹-); IF1 (- A-); IF2 (-×-); IF3 (-*-) 
of weight among all the manufactured formulation batches. It is affected by the flow properties of the powder blend. As weight of the prepared tablet was $500 \mathrm{mg}$, percentage deviation allowed was $5 \%$ as per IP. Therefore all the formulation batches passed weight variation test as the percentage deviation was found to be in the range of $0.8 \%$ to $2 \%$ (Table 4 ) for all the formulations prepared. So these results suggested that there was no significant variation in weight between different batches of tablets prepared. This showed uniform die filling during tablet compression.

Hardness gives an idea regarding how far the tablets are resistant to capping, aberration or breakage under conditions of storage, transportation and handling of the tablet. It affects drug dissolution and release to some extent. The hardness of all the formulations was in the range of $5.5-6.5 \mathrm{~kg} / \mathrm{cm}^{2}$ (Table 4). Therefore all the batches were found to show no alteration in dissolution profile and had good tablet hardness to withstand the handling abrasion.

Crushing strength alone is not an absolute indicator of tablet strength. Therefore another measure of tablet strength is friability test that gives information regarding percentage loss in weight by tablets due to mechanical stress. Friability test decides whether the prepared tablets can withstand the mechanical stress and confirms their suitability for further processing. Friability of the prepared tablets was found to be in the range of $0.421-0.616 \%$ (Table 4). As friability was below $1 \%$, indicating prepared tablets in each formulation can withstand the mechanical shocks and the moisture content of the powder blends were within limits.

Drug content estimation helps in ensuring the consistency of dosage units, in which each unit in a batch should have drug content as per the limits of IP. Assay of gastro-bilayer floating tablets were studied with the help of UV-visible spectrophotometer and the content was estimated. Drug content for all the prepared formulation batches were found in the range of $99.55-100.29 \%$ for simvastatin and $99.15-101.8 \%$ for atenolol, which were within the limits.

Ideally, least possible floating lag time and continuous flotation of the dosage form in the upper GIT by

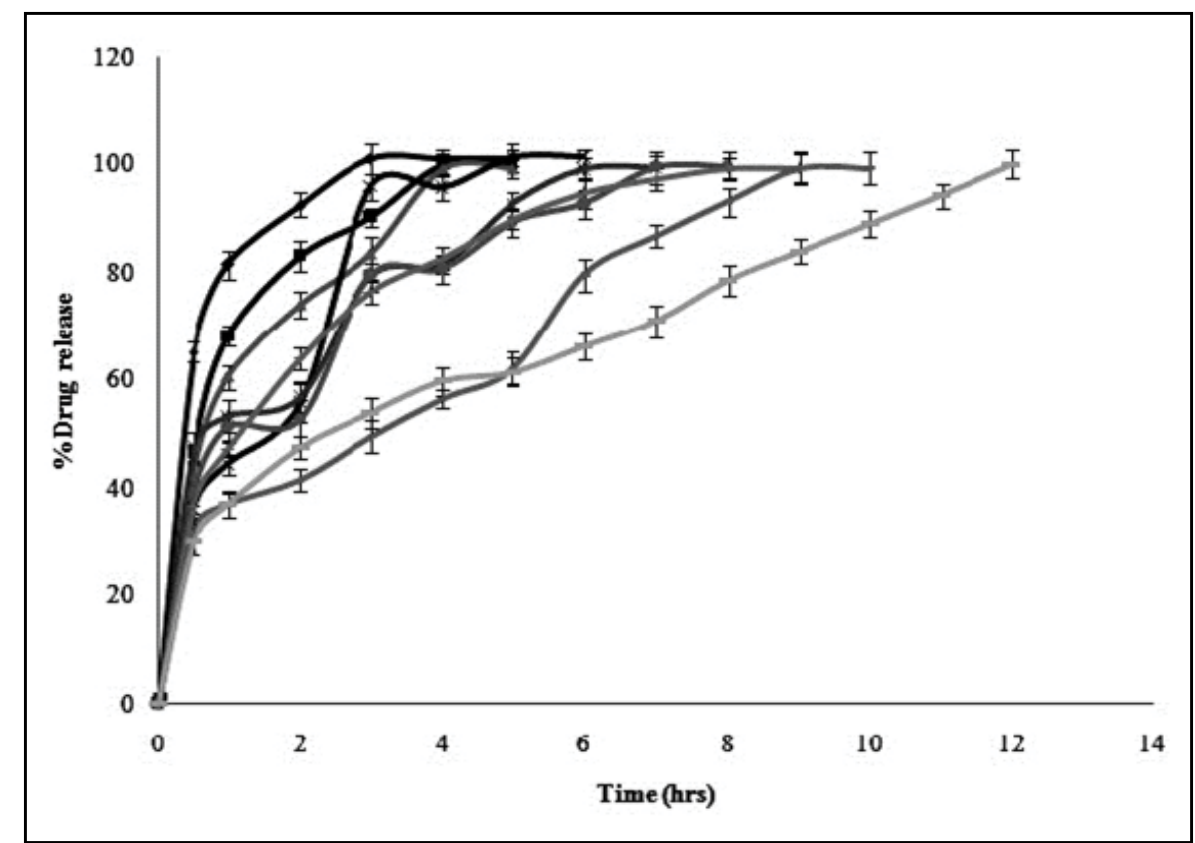

Fig. 6: In vitro release profiles of sustained release tablets.

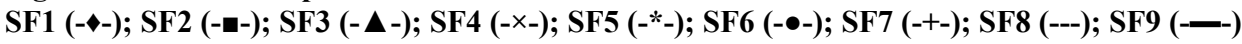

TABLE 4: POST COMPRESSION PARAMETERS OF GASTRO-BILAYER FLOATING TABLETS

\begin{tabular}{|c|c|c|c|c|c|c|c|c|}
\hline \multirow{2}{*}{$\begin{array}{l}\text { Formulation } \\
\text { code }\end{array}$} & \multirow{2}{*}{$\begin{array}{c}\text { Weight } \\
\text { variation }^{\mathrm{a}}(\mathrm{mg})\end{array}$} & \multirow{2}{*}{$\begin{array}{l}\text { Hardness }^{\mathrm{b}} \\
\left(\mathrm{kg} / \mathrm{cm}^{2}\right)\end{array}$} & \multirow{2}{*}{$\begin{array}{c}\text { Thickness }^{c} \\
(\mathrm{~mm})\end{array}$} & \multirow[t]{2}{*}{ Friability $^{d}$} & \multicolumn{2}{|c|}{ Drug content $^{\mathrm{e}}(\%)$} & \multirow{2}{*}{$\begin{array}{l}\text { FLT } \\
(\min )\end{array}$} & \multirow{2}{*}{$\begin{array}{r}\text { TFT } \\
\text { (h) }\end{array}$} \\
\hline & & & & & Atenolol & Simvastatin & & \\
\hline ISF1 & $490 \pm 2 \%$ & $6.1 \pm 0.16$ & $2.83 \pm 0.54$ & 0.604 & $99.15 \pm 1.2$ & $99.55 \pm 0.7$ & 15 & 12 \\
\hline ISF2 & $494 \pm 1.2 \%$ & $5.5 \pm 0.18$ & $2.75 \pm 0.89$ & 0.421 & $100.51 \pm 0.4$ & $100.29 \pm 0.6$ & 9 & 12 \\
\hline ISF3 & $496 \pm 0.8 \%$ & $6.5 \pm 0.08$ & $2.48 \pm 0.14$ & 0.616 & $101.8 \pm 0.6$ & $99.75 \pm 0.9$ & 7 & 8 \\
\hline
\end{tabular}

a: $A v g \pm \%$ deviation, $n=20 ;$ b: mean $\pm S D, n=6 ; c:$ mean $\pm S D, n=5 ; d: n=6.5$ g; e: $m e a n \pm S D, n=20$ 
preventing the dosage form from escaping to the lower GIT is necessary for providing local drug absorption in the stomach. Therefore the concentration of sodium bicarbonate was critical factor to arrive at the shortest lag time and to prolong the gastric retention time. This may be due to the fact that sodium bicarbonate has the ability to decrease the density of the tablets as produces $\mathrm{CO}_{2}$ bubbles on reaction with hydrochloric acid, resulting in the formation of pores due to entrapment of bubbles in the swollen polymer matrices of HPMC which ultimately helped the dosage form to float on to the surface of the medium. In addition by using HPMC persistent buoyancy was achieved. In vitro floating ability was determined by considering sodium bicarbonate in three concentrations $3.75,5$ and $6.25 \%$ w/w. It was observed that an increased concentration of sodium bicarbonate, decreased the floating lag time from 15 to $7 \mathrm{~min}$ (Table 4). Different stages of floating of the gastro-bilayer tablet were shown in fig. 7. The formulation ISF2 was considered as the good formulation as it has good floating lag time $(7 \mathrm{~min})$ along with total float time (up to $12 \mathrm{~h}$ ).

Dissolution profile of simvastatin, incorporated as an immediate release layer in the gastro-bilayer floating tablets, showed more than $95 \%$ of the drug release within $15 \mathrm{~min}$ as shown in fig. 8a. Increasing concentrations of sodium bicarbonate may produce higher level of effervescence that may result in increased rate of pore generation which in turn results in rapid hydration of the matrices and consequently faster drug release may occur $^{[28]}$. The formulation ISF1 showed incomplete drug release $(80 \%)$ of over a period of $12 \mathrm{~h}$. This may be due to decreased rate of pore formation which results in slower rate of drug release. The formulation batch ISF3 showed $101 \%$ of drug release in a period of $7 \mathrm{~h}$, it may be due to higher concentration of sodium bicarbonate that resulted in increased rate of pore formation lead to loss of integrity of the tablets which may result in faster drug release. The complete drug was release from the formulation batch ISF2 over a period of $12 \mathrm{~h}$ without losing the integrity of the tablet, considered as optimized formulation (fig. 8b).

The in vitro release data of the formulated batches were fitted into the different kinetic models to explore the drug release pattern. Optimized formulation showed biphasic release pattern i.e. burst effect followed by sustained release. Burst effect may be due to highly hydrophilic nature of drug, polymer and direct compression method that allows the drug to be on the surface of the tablet without entering into the matrix. Therefore when the tablet comes in contact with the dissolution medium, the drug present on the surface of the tablet entered into medium. Along with this free drug, after $1 \mathrm{~h}$ chain relaxation also occurs for the polymers present at the surface releasing some amount of drug into the dissolution medium (36.53\%). So burst effect is an additive effect of the free drug present at the surface and the initial release of drug from the instantly swelled gel barrier. The result further can be supported by the fact that presence of sodium bicarbonate also leads to formation of bubbles facilitating the drug release during initial stages. When

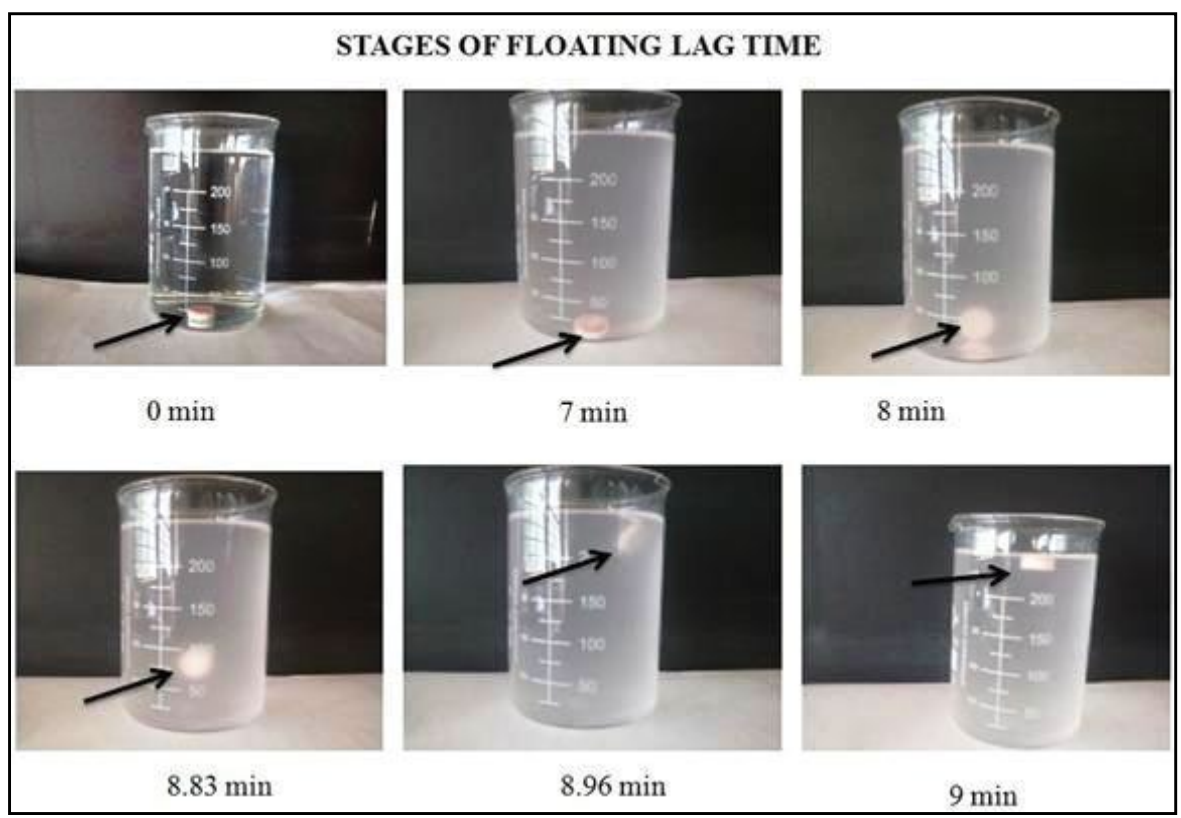

Fig. 7: Stages of floating of the prepared gastro-bilayer floating tablets. 


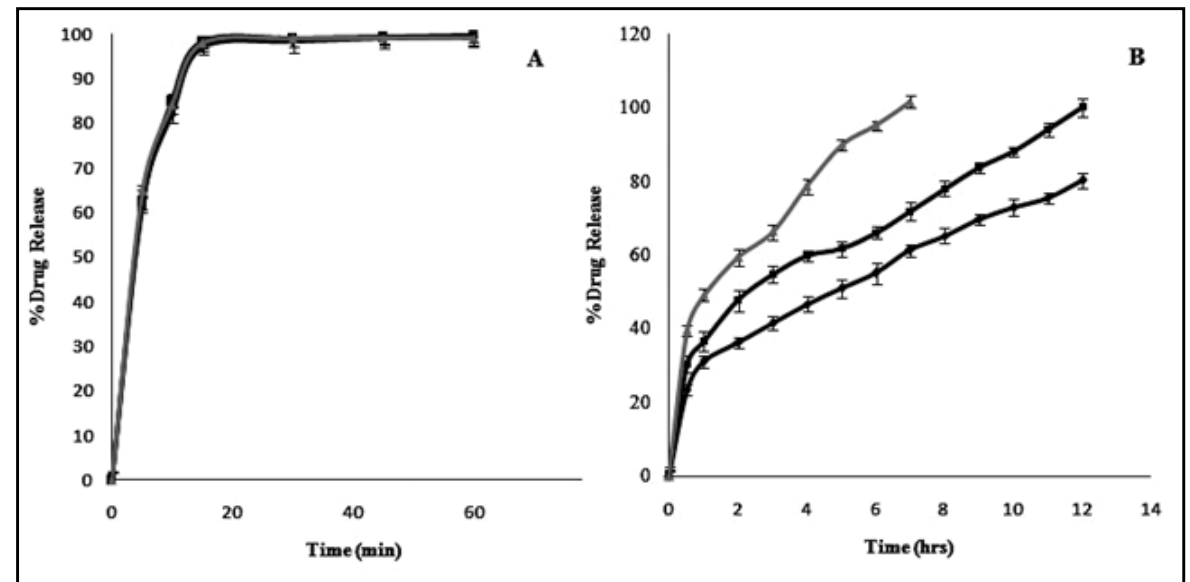

Fig. 8: In vitro dissolution profile of gastro-bilayer floating tablets.

In vitro dissolution profile of (A) IR layer (B) SR layer in gastro-bilayer floating tablets. ISF1-IR (-४-); ISF2-IR (-口-); ISF3-IR (- A-); ISF1-SR (- $\bullet$-); ISF2-SR (-ח-); ISF3-SR (- A-)

TABLE 5: RELEASE KINETICS OF GASTRO-BILAYER FLOATING TABLETS

\begin{tabular}{ccccccccccc}
\hline Formulation & \multicolumn{2}{c}{ Zero order } & \multicolumn{2}{c}{ First order } & \multicolumn{2}{c}{ Higuchi } & \multicolumn{2}{c}{ Korsmeyer and Peppas } & \multicolumn{2}{c}{ Hixson Crowell } \\
\cline { 2 - 11 } code & $\mathrm{r}^{2}$ & $\mathrm{~K}_{0}$ & $\mathrm{r}^{2}$ & $\mathrm{~K}_{1}$ & $\mathrm{r}^{2}$ & $\mathrm{~K}_{\mathrm{H}}$ & $\mathrm{r}^{2}$ & $\mathrm{~N}$ & $\mathrm{r}^{2}$ & $\mathrm{~K}$ \\
\hline ISF1 & 0.901 & 5.548 & 0.007 & 0.025 & 0.987 & 21.50 & 0.985 & 0.375 & 0.996 & -0.012 \\
ISF2 & 0.892 & 6.707 & 0.138 & 0.140 & 0.981 & 26.06 & 0.985 & 0.363 & 0.787 & -0.302 \\
ISF3 & 0.762 & 7.028 & 0.446 & 0.283 & 0.936 & 28.85 & 0.982 & 0.320 & 0.922 & -0.514 \\
\hline
\end{tabular}

the data was subjected to zero order and first order model higher $\mathrm{r}^{2}$ values was obtained for zero order (0.892) compared with first order (0.138) suggests that formulation follows zero order. This may be due to the fact that depleted zone act as reservoir matrix. The optimized formulation was studied for Higuchi and Hixson Crowell model where Higuchi model $\mathrm{r}^{2}$ value (0.981) was found to be higher than the Hixson Crowell value i.e. 0.787 (Table 5) which reveals that release may follow diffusion mechanism. This may be due to hydrophilic nature of drug incorporated in a semi-solid matrix or sodium bicarbonate that forms gas bubbles on reaction with dissolution medium which creates porous system that allows the drug to diffuse through the tortuous pathway created by the porous system. Release exponent (n) value of optimized formulation was found to be 0.363 that indicates Fickian diffusion. HPMC being a swellable polymer when it comes in contact with dissolution medium forms a gel (depletion layer model). After a period of time, the release was controlled by this gel layer which acts as a barrier for the drug to release. Drug has to pass through this barrier gel layer before entering into the dissolution medium. As the process of dissolution continues, thickness of this barrier gel layer increases so continuously drug release decreases and becomes more controlled.

\section{Acknowledgements:}

The authors would like to thank National Chemicals, Vadodara, India for supplying the pure drug simvastatin. We are also thankful to MANSAS Educational Trust and Dr. P. Uday Shankar, Principal, Maharajah's College of Pharmacy, Vizianagaram for providing required facilities to carry out this research work.

\section{Financial support and sponsorship:}

Nil.

\section{Conflicts of interest:}

There are no conflicts of interest.

\section{REFERENCES}

1. Nakagawa T, Kondo S, Sasai Y, Kuzuya M. Preparation of floating drug delivery system by plasma technique. Chem Pharm Bull 2006;54:514-8.

2. Pawar VK, Kansal S, Garg G, Awasthi R, Singodia D, Kulkarni GT. Gastroretentive dosage forms: A review with special emphasis on floating drug delivery systems. Drug Deliv 2011;18:97-10.

3. Garg R, Gupta GD. Preparation and evaluation of gastroretentive floating tablets of silymarin. Chem Pharm Bull 2009;57:545-9.

4. Guguloth M, Bomma R, Veerabrahma K. Development of sustained release floating drug delivery system for norfloxacin: in vitro and in vivo evaluation. PDA J Pharm Sci Technol 2011;65:198-206. 
5. He W, Li Y, Zhang R, Wu Z, Yin L. Gastro-floating bilayer tablets for the sustained release of metformin and immediate release of pioglitazone: Preparation and in vitro/in vivo evaluation. Int J Pharm 2014;476:223-31.

6. Abebea A, Akselib I, Sprockela O, Kottalaa N, Cuitino AM. Review of bilayer tablet technology. Int $\mathrm{J}$ Pharm 2014;461:549-58.

7. Fox CS. Cardiovascular disease risk factors, Type 2 diabetes mellitus, and the Framingham heart study. Trends Cardiovasc Med 2010;20:90-95.

8. Lavie CJ, Milani RV, Ventura HO. Obesity and cardiovascular disease risk factor, paradox, and impact of weight loss. J Am Coll Cardiol 2009;53:1925-32.

9. Department of Health Statistics and Informatics. Causes of death 2008: data sources and methods. Geneva: World Health Organization; 2008.

10. Lim SS, Vos T, Flaxman AD, Danaei G, Shibuya K, Rohani HA, et al. A comparative risk assessment of burden of disease and injury attributable to 67 risk factors and risk factor clusters in 21 regions, 1990-2010: A systematic analysis for the global burden of disease study 2010. Lancet 2012;380:2224-60.

11. Dunbar RL. Hypertension, lipoprotein abnormalities and the metabolic syndrome. In: Townsend RR, Mohler ER, editors. Advanced therapy in hypertension and vascular disease. 1st ed. United States: B.C. Decker Inc; 2006. p. 361-82.

12. Kai H, Kuwahara F, Tokuda K, Shibata R, Kusaba K, Niiyama $\mathrm{H}$, et al. Coexistence of hypercholesterolemia and hypertension impairs adventitial vascularization. Hypertension 2002;39:455-9.

13. Dey S, Mazumder B, Chattopadhyay S, Das MK, Sinha $\mathrm{S}$, Ganguly S, et al. Polymers derived from Xanthomonas campesteris and Cyamopsis tetragonolobus used as retardant materials for the formulation of sustained release floating matrix tablet of atenolol. Int J Biol Macromol 2014;65:346-56.

14. Thomas SHL. Hypertension. In: Walker R, Whittlesea C, editors. Clinical Pharmacy and Therapeutics. 4th ed. Philadelphia: Churchill Livingstone Elsevier; 2008. p. 265-79.

15. Pawar HA, Gharat PR, Dhavale RV, Joshi PR, Rakshit PP. Development and evaluation of gastroretentive floating tablets of an antihypertensive drug using hydrogenated cotton seed oil. ISRN Pharm 2013;1-9.

16. Murtaza G. Solubility enhancement of simvastatin: A review. Acta Pol Pharm 2012;69:581-90.
17. Schachter M. Chemical, pharmacokinetic and pharmacodynamic properties of statins: An update. Fundam Clin Pharmacol 2005;19:117-25.

18. Kulkarni A, Bhatia M. Development and evaluation of regioselective bilayer floating tablets of atenolol and lovastatin for biphasic release profile. Iran J Pharm Res 2009;8:15-25.

19. Bolourchian N, Mahboobiana MM, Dadashzadeh S. The effect of PEG molecular weights on dissolution behaviour of simvastatin in solid dispersions. Iran $\mathrm{J}$ Pharma Res 2013;12:11-20.

20. Jun SW, Kim MS, Kim JS, Park HJ, Lee S, Woo JS, et al. Preparation and characterization of simvastatin/ hydroxypropyl- $\beta$-cyclodextrin inclusion complex using supercritical antisolvent (SAS) process. Eur J Pharm Biopharm 2007;6:413-21.

21. Newa M, Bhandari KH, Li DX, Kwon TH, Kim JA, Yoo BK, et al. Preparation, characterization and in vivo evaluation of ibuprofen binary solid dispersions with $\mathrm{p} 188$. Int J Pharm 2007;343:228-37.

22. Rosa M, Zia H, Rhodes T. Dosing and testing in vitro of a bioadhesive and floating drug delivery system for oral application. Int J Pharm 1994;105:65-70.

23. Higuchi $T$. Kinetic analysis of in vitro release rates of zidovudine matrix tablets. Theoretical analysis of rate release of solid drugs dispersed in solid matrices. J Pharm Sci 1963;52:1145-9.

24. Korsemeyer RW, Gurny R, Doelker E, Buri P, Peppas NA. Kinetic analysis of in vitro release of zidovudine matrix tablets. Mechanism of solute release from porous hydrophilic polymers. Int J Pharm Sci 1983;4:25-35.

25. Robinson JR, Eriksen SP. Theoretical formulation of sustained release dosage forms. J Pharm Sci 1966;55:1254-63.

26. Pandya P, Gattani S, Jain P, Khirwal L, Surana S. Co-solvent evaporation method for enhancement of solubility and dissolution rate of poorly aqueous soluble drug simvastatin: in vitro-in vivo evaluation. AAPS PharmSciTech 2008;9:1247-52.

27. Yin L, Qin C, Chen K, Zhu C, Cao H, Zhou J, et al. Gastro floating tablets of cephalexin: Preparation and in vitro/in vivo evaluation. Int J Pharm 2013;452:241-48.

28. Trados MI. Controlled release effervescent floating matrix tablets of ciprofloxacin hydrochloride: Development, optimization and in vitro-in vivo evaluation in healthy volunteers. Eu J Pharm Biopharm 2010;74:332-9. 